\title{
Dynamic Groups with inbuilt data updating for loud and Anti Collusion Data Sharing Scheme
}

\author{
M. Nandhini Sri ${ }^{1}$, R. Sangeetha ${ }^{2}$, Mr. S. Radhakrishnan ${ }^{3}$ \\ Bachelor of Technology in Information Technology, Kamaraj college of Engineering and Technology, Virudhunagar ${ }^{1,2}$ \\ Assistant Professor/IT, Kamaraj college of Engineering and Technology, Virudhunagar ${ }^{3}$
}

\begin{abstract}
The Benefited from Cloud Computing, clients can achieve a flourishing and moderate methodology for information sharing among gathering individuals in the cloud with the characters of low upkeep and little administration cost. Then, security certifications to the sharing information records will be given since they are outsourced. Horribly, due to the never-ending change of the enrolment, sharing information while giving protection saving is still a testing issue, particularly for an untrusted cloud because of the agreement attack. In addition, for existing plans, the security of key dispersion depends on the safe communication channel, then again, to have such channel is a solid feeling and is difficult for practice. In this paper, we propose a safe information sharing plan for element individuals. Firstly, we propose a safe route for key dispersion with no safe correspondence channels, and the clients can safely acquire their private keys from gathering administrator. Besides, the plan can accomplish fine-grained access control, any client in the gathering can utilize the source in the cloud and refused clients can't get to the cloud again after they are rejected. Thirdly, we can protect the plan from trickery attack, which implies that rejected clients can't get the first information record regardless of the possibility that they scheme with the untrusted cloud. In this methodology, by utilizing polynomial capacity, we can achieve a protected client denial plan. At long last, our plan can bring about fine productivity, which implies past clients need not to overhaul their private keys for the circumstance either another client joins in the gathering or a client is give up from the gathering.
\end{abstract}

Keywords: Access control, Privacy-preserving, Key distribution, Cloud computing.

\section{INTRODUCTION}

Cloud Computing, with the characteristics of natural information sharing and low support, gives a superior usage of resources. In Cloud Computing, cloud administration suppliers offer a reflection of boundless storage room for customers to host information [1]. It can offer customers some support with reducing their money related overhead of information administrations by moving the nearby administrations framework into cloud servers.however, security concerns turn into the principle control as we now outsource the capacity of information, which is perhaps delicate, to cloud suppliers. To safeguard information security, a typical methodology is to encode information records before the customers transfer the scrambled information into the cloud [2]. Unfortunately, it is hard to outline a protected and productive information sharing plan, particularly for element groups in the cloud.

Kallahalla et al [3] displayed a cryptographic supply framework that empowers secure information sharing on untrust servers taking into account the procedures that isolating documents into filegroups and scrambling each file_group with a record square key. In any case, the record square keys should be upgraded and circulated for a client denial, along these lines, the framework had a extensive key appropriation overhead. Different plans for information sharing on untrusted servers have been proposed. [4],[5]. As it might, the complexities of client interest and renouncement in these plans are straightly expanding with the quantity of information owner and the repudiated clients. Yu et al [6] altered and joined procedures of key strategy trait based encryption [7], intermediary re-encryption and slow re-encryption to accomplish fine-grained information access control without presentation information substance. Be that as it may, the single-proprietor way might block the usage of uses, where anypart in the gathering can utilize the cloud administration to store and impart information records to others. Lu et al [8] proposed a protected origin plan by utilizing bunch marks and cipher text-arrangement characteristic based encryption methods [9]. Every client gets two keys after the recruitment while the assign key is utilized to decode the information which is scrambled by the quality based encryption and the gathering mark key is make use for security protecting and traceability. Then again, the denial is not upheld in this plan. Liu et al [10] exhibited a protected multi-proprietor information sharing plan, named Mona. It is guaranteed that the plan can achieve fine-grained access control and renounced clients won't have the capacity to get to the sharing information again once they are disavowed. In any case, the plan will naturally experience the ill effects of the plot attack by the repudiated client and the cloud [13]. The disavowed client can utilize his private key to decode the encoded information record and get the secrecy information after his denial by plotting with the cloud. In the period of 
document access, as a matter of first importance, the renounced client sends his solicitation to the cloud, then the cloud responds the relating scrambled information record and denial rundown to the repudiated client without checks. Next, the renounced client can figure the decoding key with the assistance of the assault calculation. At last, this assault can prompt the renounced clients getting the sharing information and uncovering different secrecy of honest to goodness individuals. Zhou et al [14] displayed a safe access control plan on scrambled information in distributed storage by summoning part based encryption method. It is guaranteed that the plan can accomplish creative client denial that joins part based access control approaches with encryption to secure wide information supply in the cloud.

Unfortunately, the confirmations between elements are not concerned, the plan effortlessly experience the ill effects of assaults, for instance, conspiracy assault. At last, this assault can prompt enlightening touchy information documents. Zou et al. [15] displayed a down to earth and adaptable key administration system for trusted cooperative registering.

By utilizing access control polynomial, it is intended to accomplish proficient access control for element bunches. unfortunately, the protected path for sharing the individual changeless flexible mystery between the client and the server is not encouraged and the private key will be revealed once the individual continuous convenient mystery is acquired by the attackers. In this paper, we propose a protected information sharing plan, which can achieve secure key requisition and information sharing for element bunch.

The principle commitments of our plan include:

1) We give a safe approach to key transport with no protected correspondence channels. The clients can safely obtain their private keys from gathering chief with no Certificate Authorities because of the confirmation for people in general key of the client.

2) Our plan can accomplish fine-grained access control, with the assistance of the gathering client list, any client in the gathering can make use of the source in the cloud and disavowed clients can't get to the cloud again after they are denied.

3) We propose a safe information sharing plan which can be protected from agreement attack. The denied clients can not have the capacity to get the first information records once they are rejected regardless of the fact that they contrive with the untrusted cloud. Our plan can accomplish secure client rejection with the assistance of polynomial capacity.

4) Our plan can encourage dynamic gatherings effectively, when another client joins in the gathering or a client is renounced from the gathering, the private keys of alternate clients don't should be recomputed and renovate.

5) Security investigation to demonstrate the security of our plan. In expansion,Performance of re-enhancement to exhibit the effectiveness of our plan.

\section{RELATED WORKS}

[1] We provide a secure way for key distribution without any secure communication channels. The users can securely obtain their private keys from group manager without any Certificate Authorities due to the verification for the public key of the user.

[2] Our scheme can achieve fine-grained access control, with the help of the group user list, any user in the group can use the source in the cloud and revoked users cannot access the cloud again after they are revoked.

[3] We propose a secure data sharing scheme which can be protected from collusion attack. The revoked users can not be able to get the original data files once they are revoked even if they conspire with the untrusted cloud. Our scheme can achieve secure user revocation with the help of polynomial function.

[4] Our scheme is able to support dynamic groups efficiently, when a new user joins in the group or a user is revoked from the group, the private keys of the other users do not need to be recomputed and updated.

[5].We provide security analysis to prove the security of our scheme. In addition, we also perform simulations to demonstrate the efficiency of our scheme.

\section{PROPOSED SYSYTEM}

In this paper, we propose a secure data sharing scheme, which can achieve secure key distribution and data sharing for dynamic group. We provide a secure way for key distribution without any secure communication channels. The users can securely obtain their private keys from group manager without any Certificate Authorities due to the verification for the public key of the user. Our scheme can achieve fine-grained access control, with the help of the group user list, any user in the group can use the source in the cloud and revoked users cannot access the cloud again after they are revoked. We propose a secure data sharing scheme which can be protected from collusion attack. The revoked users can not be able to get the original data files once they are revoked even if they conspire with the untrusted cloud.

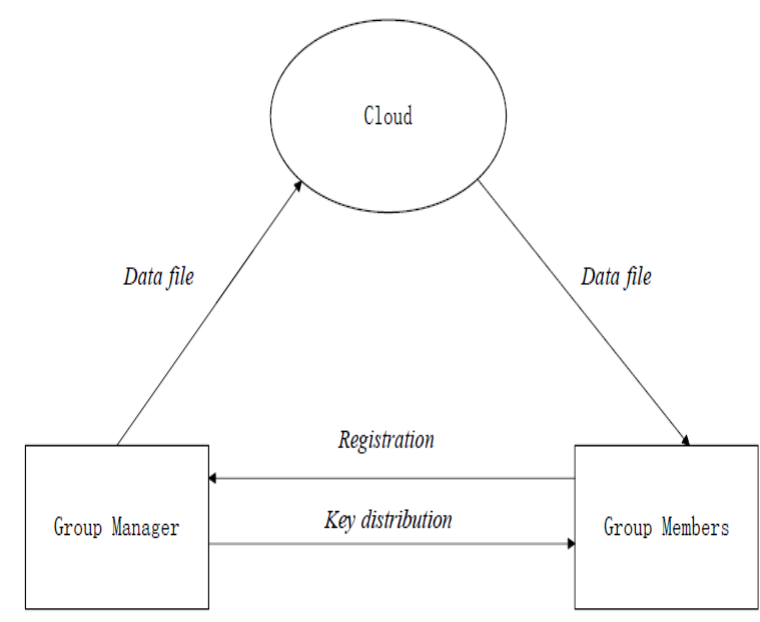


Our scheme can achieve secure user revocation with the help of polynomial function. Our scheme is able to support dynamic groups efficiently, when a new user joins in the group or a user is revoked from the group, the private keys of the other users do not need to be recomputed and updated. We provide security analysis to prove the security of our scheme

\section{RESULT}

We design a secure anti-collusion data sharing scheme for dynamic groups in the cloud. In our scheme, the users can securely obtain their private keys from group manager Certificate Authorities and secure

Communication channels. Also, our scheme is able to support dynamic groups efficiently, when a new user joins in the group or a user is revoked from the group, the private keys of the other users do not need to be recomputed and updated. After uploading the file the user can able to update their file within the product itself, no need of updation in the system Moreover, our scheme can achieve secure user revocation; the revoked users can not be able to get the original data files once they are revoked even if they conspire with the untrusted cloud.

\section{CONCLUSION}

In this paper, we outline a protected against agreement information sharing plan for element bunches in the cloud. In our plan, the clients can safely acquire their private keys from gathering director Certificate Authorities and secure correspondence channels. Likewise, our plan can bolster dynamic gatherings proficiently, when another client joins in the gathering or a client is denied from the gathering, the private keys of alternate clients don't should be recomputed and redesigned. In addition, our plan can accomplish secure client repudiation, the disavowed clients can not have the capacity to get the first information records once they are denied regardless of the possibility that they plot with the untrusted cloud

\section{FUTURE ENHANCEMENT}

In this research work, we have reviewed to provide a secure environment where a data owner can share data with members of his group while preventing any outsiders from gaining any data access in case of malicious activities such as data loss and theft. However, throughout this work we assume that members of the group will not carry out malicious activities on the data owner's data.

Auditing and Accountability in the Cloud is a potential for future research in the context of data sharing in the Cloud. Many users in particular organizations and enterprises gain the benefit from data sharing in the Cloud.

However, there is always a likely chance that members of the group can carry out illegal operations on the data such as making illegal copies and distributing copies to friends, general public, etc in order to profit. A future research direction would be to find ways for a data owner to hold accountable any member that carries out malicious activities on their data.

\section{REFERENCES}

[1] M. Armbrust, A. Fox, R. Griffith, A. D. Joseph, R. Katz, A. Konwinski, G. Lee, D. Patterson, A. Rabkin, I. Stoica, and M. Zaharia,"A view of cloud computing," Commun. ACM, vol. 53, no. 4, pp. 50-58, Apr. 2010.

[2] S. Kamara and K. Lauter, "Cryptographic cloud storage," in Proc.Int. Conf. Financial Cryptography Data Security, Jan. 2010, pp. 136-149.

[3] M. Kallahalla, E. Riedel, R. Swaminathan, Q. Wang, and K. Fu, "Plutus: Scalable secure file sharing on untrusted storage," in Proc. USENIX Conf. File Storage Technol., 2003, pp. 29-42.

[4] E. Goh, H. Shacham, N. Modadugu, and D. Boneh, "Sirius: Securing remote untrusted storage," in Proc. Netw. Distrib. Syst. Security Symp., 2003, pp. 131-145.

[5] G. Ateniese, K. Fu, M. Green, and S. Hohenberger, "Improved proxy re-encryption schemes with applications to secure distributed storage," in Proc. Netw. Distrib. Syst. Security Symp., 2005, pp. 29-43.

[6] Shucheng Yu, Cong Wang, Kui Ren, and Weijing Lou, "Achieving Secure, Scalable, and Fine-grained Data Access Control in Cloud Computing," Proc. ACM Symp. Information, Computer and Comm. Security, pp. 282-292, 2010.

[7] V. Goyal, O. Pandey, A. Sahai, and B. Waters, "Attribute-Based Encryption for Fine-Grained Access Control of Encrypted Data," Proc. ACM Conf. Computer and Comm. Security (CCS), pp. 89-98, 2006

[8] R. Lu, X. Lin, X. Liang, and X. Shen, "Secure Provenance: The Essential of Bread and Butter of Data Forensics in Cloud Computing," Proc. ACM Symp. Information, Computer and Comm. Security, pp. 282-292, 2010. 